DOI: https://doi.org/10.46296/yc.v4i7.0046

\title{
EVALUACIÓN DE LA CALIDAD DEL PROCESO DE NIVELACIÓN DE LA CARRERA DE ADMINISTRACIÓN DE EMPRESAS EN LA UNIVERSIDAD TÉCNICA DE MANABÍ, ECUADOR.
}

\section{EVALUATION OF THE QUALITY OF THE LEVELING PROCESS OF THE BUSINESS ADMINISTRATION CAREER AT THE TECHNICAL UNIVERSITY OF MANABÍ, ECUADOR.}

\author{
Cantos-Zambrano José Roberto ${ }^{1 *}$; Ramos-Alfonso Yanelis ${ }^{2}$ \\ ${ }^{1}$ Maestrante del programa de posgrado Gerencia de la Calidad e Innovación, Universidad \\ Técnica de Manabí, UTM. Portoviejo, Ecuador. \\ ${ }^{2}$ Docente de la Universidad Técnica de Manabí, UTM. Portoviejo, Ecuador.
}

*Correo: jcantos1099@utm.edu.ec

\begin{abstract}
Resumen
La calidad en la educación superior tiene un rol esencial, por cuanto se trata de asegurar que los estudiantes tengan una preparación que responda a sus expectativas, teniendo un impacto positivo en el proceso académico y en su posterior inserción laboral, por ello el Gobierno Central, a través de los órganos competentes, ha establecido las estrategias y normas de Aseguramiento de la Calidad Educativa. El objetivo del presente trabajo se centró en evaluar la percepción de la calidad del proceso de Nivelación del Centro de Promoción y Apoyo al Ingreso (CPAI) de la carrera de Administración de empresas de la Universidad Técnica de Manabí. Para tal efecto, se aplicó el modelo SERVQUAL, específicamente la diferencia 5 referida a la satisfacción de estudiantes y la diferencia 1 desde un enfoque del conocimiento de las percepciones estudiantiles, por parte de los directivos. Se obtuvo una moda de 4 de forma general, lo que establece una adecuada satisfacción de los estudiantes. Se manifestaron oportunidades de mejora a través de las diferencias de los criterios de directivos y estudiantes en cuanto a empatía y capacidad de respuesta, debido a limitaciones en la comunicación, estabilidad de las plataformas y estandarización de los procesos; elementos a considerarse para la mejora continua de la calidad en el proceso estudiado.
\end{abstract}

Palabras clave: calidad, satisfacción, educación superior, expectativas, SERVQUAL.

\begin{abstract}
Quality in higher education plays an essential role, since it is about ensuring that students have a preparation that responds to their expectations, having a positive impact on the academic process and on their subsequent job placement, therefore the Central Government, through the competent bodies, it has established the strategies and standards for the Assurance of Educational Quality. The objective of this work was focused on evaluating the perception of the quality of the Leveling process of the Center for Promotion and Support for Income (CPAI) of the Business Administration career of the Technical University of Manabí. For this purpose, the SERVQUAL model was applied, specifically difference 5 referring to student satisfaction and difference 1 from an approach to knowledge of student perceptions by managers. For this purpose, the SERVQUAL model was applied, specifically difference 5 referring to student satisfaction and difference 1 from an approach to knowledge of student perceptions by managers. A mode of 4 was obtained in general, which establishes adequate student satisfaction. Opportunities for improvement were manifested through the differences in the criteria of managers and students in terms of empathy and response capacity, due to limitations in communication, stability of platforms and standardization of processes; elements to be considered for the continuous improvement of quality in the studied process.
\end{abstract}

Keywords: quality, satisfaction, higher education, expectations, SERVQUAL.

Información del manuscrito:

Fecha de recepción: 04 de mayo de 2020.

Fecha de aceptación: 09 de julio de 2020.

Fecha de publicación: 10 de julio de 2020. 


\section{Introducción}

Hoy en día, el término calidad se ha adherido a todo tipo de prácticas empresariales, laborales y del sector educativo. En tal sentido, el concepto de calidad es una construcción social, que varía según los intereses de los grupos internos y externos de una institución educativa, que refleja las características de la sociedad que se desea hoy y se proyecta para el futuro. No es un concepto unívoco y fijo, sino que debe ser construido a través de concesos y negociaciones entre los actores (Días, 1995, citado en (Pesantez, 2011).

Así también este término es definido por la (Organización de las Naciones Unidas para la Educación, la Ciencia y la Cultura [UNESCO], 1996) como intrínseca a la actividad del ser, así "La preocupación por la calidad de la educación superior aparece vinculada a la adecuación del ser y quehacer de la misma" (UNESCO, 1998).

Al hablar de calidad en el contexto universitario, significa que éste nace al interior de la institución y requiere su aplicación en todos los procesos, para lo cual la gestión interna debe ser eficiente, de manera que pueda ofrecerse calidad en los servicios de educación superior. "La calidad en la gestión universitaria requiere su aplicación en todo momento, desde el control del trabajo y del talento humano que posee, hasta la dirección eficiente de los esfuerzos comunes para la consecución de metas y objetivos académicos" (Cárdenas, 2016).

En Latinoamérica, los mecanismos para el aseguramiento y acreditación de la calidad de universidades son escasos. Las universidades de origen extranjero que operan en países de la región asumen en general uno de dos caminos: o se registran como universidad dentro del país siguiendo los procedimientos establecidos para su autorización, control y vigilancia como una universidad local o se asocian con una universidad local para otorgar los diplomas, grados $y$ títulos con el amparo legal de dicha universidad local. El presupuesto promedio en Latinoamérica para la educación superior es inferior al $1,5 \%$ del PIB, lo cual es evidentemente insuficiente para atender las necesidades de un sistema en desarrollo (Sáinz \& Barberá, 2019).

Una de las formas de asegurar la calidad en las Instituciones de 
educación superior es el proceso de evaluación institucional, así este:

Como instrumento de mejora, debe quedar plasmado en estructuras de significado particulares para contextos específicos, pues si no se reconocen sus especificidades difícilmente se pueda hacer alusión correctamente a las premisas de mejora continua a las cuales se aspira en la actualidad para las IES. Por ello, es necesario una cultura de autoevaluación permanente para el cumplimiento de los estándares de calidad, unido a un fuerte compromiso con el proceso formativo que se realiza en las universidades. (Sánchez, Chávez, \& Mendoza, 2018).

El informe de la (Organización de Estados Iberoamericanos para la Educación, la Ciencia y la Cultura [OEl], 2018, citado por Sáinz \& Barberá, 2019) refiere que "La responsabilidad fundamental de la calidad debe recaer en las instituciones de educación superior. Deben desarrollarse herramientas para que les ayuden en la implantación de una "cultura de calidad' efectiva en todos sus procesos.

En el Ecuador, desde el año 2012 hasta el 2016 el proceso de admisión a las Instituciones de educación superior (IES), universidades, institutos superiores tecnológicos y conservatorios, consideraba la aprobación del Examen Nacional para la Educación Superior (ENES), estandarizado, diseñado y aplicado por el Sistema Nacional de Nivelación y Admisión (SNNA), actualmente denominado Ser Bachiller, cuya responsabilidad corresponde conjuntamente al Ministerio de Educación Intercultural (MINEDUC) y a la Secretaría de Educación Superior, Ciencia, Tecnología e Innovación (SENESCYT) (Delgado, Santillán, Japón, \& Mora, 2018).

La SENESCYT es el ente regulador del proceso de Nivelación en las Instituciones de Educación Superior y otorga la facultad a las IES de decidir si ofertan cupos para nivelación de carrera, considerando la heterogeneidad en la formación de bachillerato y observando las características de las carreras universitarias. (SENESCYT, 2019).

Dentro de este proceso, los estudiantes que finalizaban la educación media (bachillerato) y querían continuar sus estudios debían rendir y alcanzar una nota mínima de 601 puntos para poder postular y ser admitidos en función de los cupos ofertados por las 
Instituciones de educación superior (IES). Este aspecto se ha convertido en un tema de debate en el que se examinan aspectos positivos y negativos. Sin embargo, es escasa la evidencia empírica sobre el proceso de admisión, desde la percepción de los aspirantes (Delgado, Santillán, Japón, \& Mora, 2018).

En ese sentido, se impone la necesidad de la evaluación de la satisfacción de los clientes de este importante proceso, con el fin de establecer estrategias para la mejora continua de la calidad percibida de los aspirantes vinculados.

Dentro de los modelos utilizados para este fin, el SERVQUAL es una técnica de investigación comercial, que permite realizar la medición de la calidad del servicio, conocer las expectativas de los clientes, y sus percepciones. (Matsumoto, 2014)

Es uno de los métodos más utilizados para medir la calidad y con base en los resultados estar en capacidad de proponer acciones de mejora, de acuerdo con el nivel de brechas o deficiencias encontradas con respecto a las percepciones y las expectativas. De acuerdo con lo expuesto por Bustamante, Zerda, Obando \& Tello (2019) en este modelo se generan 22 declaraciones basadas o resumidas en 5 atributos:

Tangibilidad: Apariencia de las instalaciones físicas, el equipo, el personal y los materiales escritos

- Fiabilidad: Capacidad para desempeñar el servicio que se promete, de manera segura y precisa.

Capacidad de respuesta: Disponibilidad para ayudar a los clientes y para promover el servicio con prontitud.

- Seguridad: Conocimiento y cortesía de los empleados y su habilidad para inspirar buena fe y confianza.

- $\quad$ Empatía: Brindar a los clientes una atención individualizada y cuidadosa.

Este conjunto de atributos ayuda a identificar las deficiencias o brechas que se generan entre lo que el cliente espera y lo que recibe (Basantes, 2017), siendo así que, se asocian a 5 tipos de brechas:

Brecha 1: Expectativas de los
clientes en relación con la
percepción de la Gerencia
gerencia y especificaciones 
- $\quad$ Brecha 3: Especificaciones de la calidad y entrega del servicio

- $\quad$ Brecha 4: Entrega del servicio y comunicación externa

- Brecha 5: Servicio esperado en relación con el servicio percibido

Este modelo de evaluación de la calidad, según lo expuesto por Barahona \& Ferrada (2012) presenta varios beneficios, tales como: información detallada sobre opiniones de clientes con respecto al servicio, niveles de desempeño, comentarios y sugerencias. También tiene limitaciones como la objetividad con que deben de ser estructuradas las preguntas y la presunción de que las necesidades de los clientes pueden documentarse y permanecer estables durante todo el proceso. No obstante, es un modelo que facilita el análisis de las percepciones en cuanto a servicios, lo cual ha sido destacado en varios estudios académicos con carácter científico, presentando al final resultados que aportan a mejorar el aseguramiento de la calidad.

Esta investigación se realiza en la Universidad Técnica de Manabí, primera universidad pública de la comunidad manabita, la que ostenta indicadores de excelencia, manifiestos en la calidad de su claustro, el alcance de su gestión por el importante número de ofertas de programas de posgrado y carreras de pregrado, el significativo número de estudiantes vinculados; entre otros elementos que la prestigian en la provincia y el país. La misma tiene como objetivo evaluar la calidad del proceso de nivelación de la carrera de Administración de Empresas, apoyado del Modelo SERVQUAL antes enunciado, como base para la mejora continua.

\section{Metodología}

El estudio se llevó a cabo en el Centro de Admisión y Apoyo al Ingreso (CPAI) de la Universidad Técnica de Manabí (UTM), ubicada en el cantón Portoviejo, provincia de Manabí. La investigación tuvo un enfoque cualitativo-cuantitativo. El diseño es no experimental y transversal, se utilizaron técnicas de recolección de datos como encuestas y entrevistas, realizadas en el semestre primero (S1) del año 2020.

La muestra objeto de estudio, en cuanto a estudiantes, estuvo 
constituida por 69 estudiantes de la carrera de Administración de Empresas, por ser una carrera de gran utilidad para la región y con una matrícula de estudiantes representativa históricamente. El error real de la investigación fue de un $9,36 \%$ lo que permite la obtención de resultados conclusivos, considerando un muestreo aleatorio simple. Al equipo Directivo se aplicó al $100 \%$ de la población. La aplicación se realizó a través de una encuesta desarrollada en Google Form. Para el análisis y procesamiento de la información recolectada, se consideraron las siguientes fases:

Fase 1. Evaluación de la calidad por los estudiantes

Se aplicó la encuesta correspondiente a la diferencia 5 del modelo SERVQUAL, utilizando una escala diferencial, obligando al encuestado a comparar entre expectativas y percepciones en los rangos siguientes: Mucho menos de lo esperado, menos de lo esperado, igual a lo esperado, más de lo esperado y mucho más de lo esperado. Los atributos analizados fueron la fiabilidad, tangibilidad, capacidad de respuesta, seguridad y la empatía. En base a los cuales pudieran establecerse las limitaciones que se presentaron en el Curso de Nivelación del CPAI en el periodo indicado, producto del confinamiento generado por el Covid-19. La medida de tendencia central utilizada fue la moda por variable y la media por atributo.

Se agregaron al final de la encuesta preguntas abiertas que motivaran a los estudiantes a proponer sugerencias para incrementar la calidad del proceso de nivelación, en un momento marcado por la emergencia mundial. Dichos resultados fueron analizados $y$ sintetizados en un diagrama de Ishikawa, que permitiera ilustrar las casusas raíces de los problemas identificados.

\section{Fase 2. Evaluación del conocimiento de los directivos de las percepciones de los estudiantes}

En esta fase se realizó un análisis similar al propuesto en la diferencia 1 del modelo Servqual, pero enfocado al conocimiento de los directivos de las percepciones, es decir, se realiza un análisis 
comparativo entre las percepciones presentadas por los estudiantes en la fase 1 y lo que los Directivos del CPAI consideran que ofrecen en cuanto a la prestación de sus servicios. Para ello se aplicó a los directivos la diferencia 1 del Modelo Servqual, enfocado a lo que estos consideran ofrecer, es decir, la calidad percibida del servicio de nivelación. Del mismo modo, se utilizó la moda para la evaluación de las variables (ítems) de la encuesta y la media por atributo. Se realizó un análisis comparativo de las medias obtenidas por atributos, para establecer las brechas entre el criterio de los directivos y los estudiantes y así identificar aquellos elementos a los que se debe prestar más atención para un mejor desarrollo de la nivelación.

\section{Fase 3. Análisis causas como base para la mejora}

En consideración a los ítems con oportunidades de mejora y las diferencias entre la percepción del servicio que se ofrece por los directivos y estudiantes. Se aplicó el diagrama de Ishikawa o de causa y efecto, que permitió el análisis de las diferencias encontradas y cómo éstas podrían convertirse en soluciones aplicables para mejorar la percepción de los servicios de Nivelación.

\section{Resultados y discusión}

\section{Fase 1. Evaluación de la calidad por los estudiantes}

se utilizó la escala de Likert de 5 categorías, ya que de acuerdo con lo expuesto por Matas (2018) constituye uno de los instrumentos más utilizados en Ciencias Sociales y estudios de mercado, de tipo diferencial, motivando a los encuestados a generar una comparación entre sus expectativas y percepciones.

La tabla 1 muestra los resultados a través de las distintas variables y atributos propuestos por el modelo SERVQUAL, reflejando que los estudiantes se encuentran satisfechos con el servicio de Nivelación, puesto que como se puede evidenciar los valores modales están por encima de los 4,00 puntos en todos los casos, lo cual equivale a "de acuerdo" y "totalmente de acuerdo". 
Tabla 1. Valores medios por variables

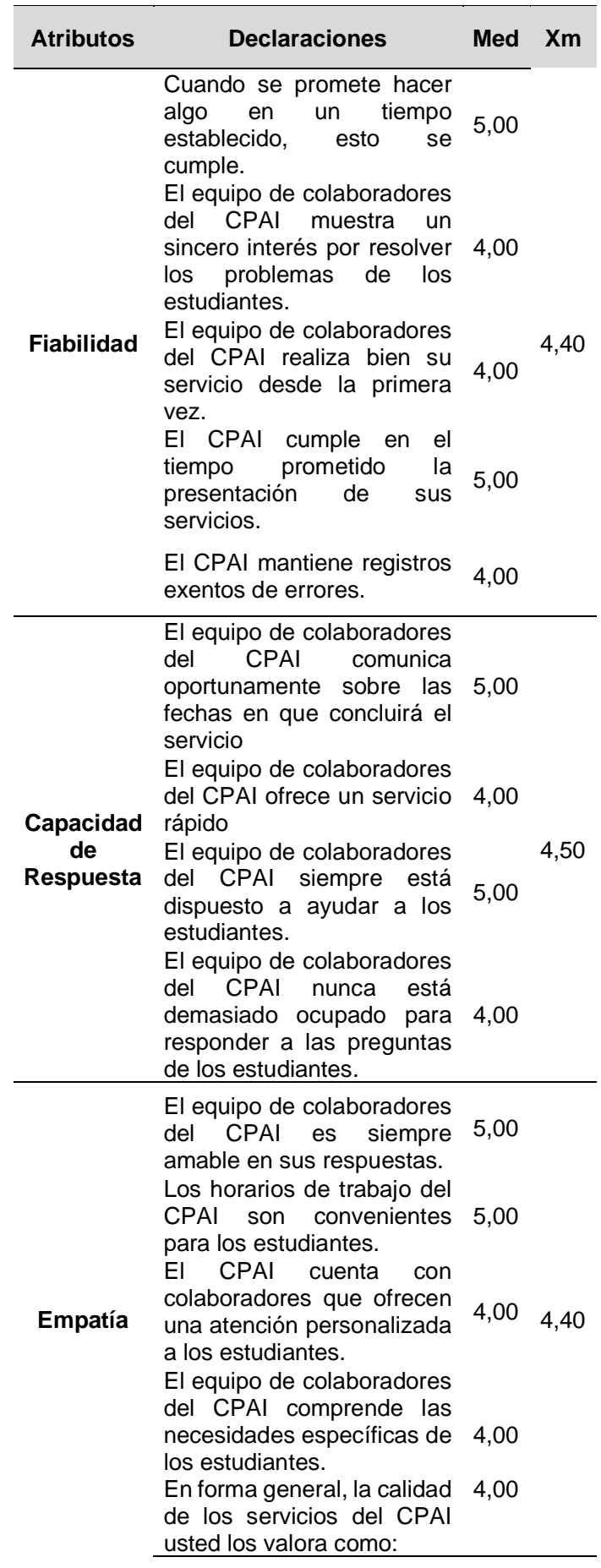

Fuente: Resultados de las encuestas aplicadas a los estudiantes

Elaborado por: Autoría propia.

Es importante resaltar, que la evaluación responde a un momento histórico de confinamiento, en el que se adoptó la modalidad virtual, producto de la pandemia provocada por el covid-19, ante lo cual fue imprescindible observar la opinión de los estudiantes con respecto a la fiabilidad de la información y el tiempo de entrega de la misma, a través de plataformas virtuales como classroom o moodle y de redes sociales como Telegram. La capacidad de respuesta tuvo un papel relevante para el análisis de la calidad del servicio. En ese sentido, la mayoría de los estudiantes se sintieron atendidos de forma oportuna y precisa, pese a las condiciones en las que se desarrolló el servicio. La empatía reflejó el nivel de compromiso por parte del equipo del CPAI para con los estudiantes, pues así lo muestran los resultados obtenidos en la tabla 1.

La moda de la pregunta de control, que responde a la calidad percibida de forma general con el servicio, fue de 4,00 puntos, lo que argumenta los resultados mencionados anteriormente, ya que como indican Álvarez, Chaparro \& Reyes (2014) los estudiantes son los que mejor pueden evaluar la calidad de los servicios educativos.

Pese a los resultados anteriores, se identificaron reservas de mejora en los comentarios de los estudiantes, 
ante las preguntas abiertas en los aspectos relacionados a la comunicación, la estabilidad de las plataformas, la estandarización y socialización de los procesos, entre otros elementos que se abordan en la Fase 3.

Fase 2. Evaluación del conocimiento de los directivos de las percepciones de los estudiantes

Luego de aplicar la encuesta a los directivos para evaluar su percepción sobre lo que reciben los estudiantes, se procedió a establecer la comparación entre ambos resultados, como se puede apreciar en la tabla 2, para el caso de los atributos más diferenciados. En términos generales, no existen diferencias significativas, pues básicamente la tendencia de percepción de calidad en el servicio se mantiene, tanto en estudiantes como en directivos. Sin embargo, se aprecia una ligera sobrevaloración de los directivos en los atributos empatía y capacidad de respuesta. A juicio de Torche, Martínez, Madrid \& Araya (2015) la dimensión subjetiva del concepto de educación de calidad puede tener diversas percepciones de acuerdo con los actores educativos analizados.

Tabla 2. Brechas promedio entre la percepción de estudiantes y directivos.

\begin{tabular}{lccc}
\hline Atributos & Estudiantes & Directivos & Brechas \\
\hline Fiabilidad & 4,40 & 4,30 & 0,10 \\
$\begin{array}{c}\text { Capacidad } \\
\text { de }\end{array}$ & 4,50 & 4,75 & $-0,25$ \\
respuesta & 4,40 & 5,00 & $-0,60$ \\
Empatía & 4 & &
\end{tabular}

Fuente: Resultados de las encuestas aplicadas a los directivos

Elaborado por: Autoría propia

Las brechas establecidas reflejan discrepancias en aspectos mínimos, como es el caso de la fiabilidad, donde los Directivos reconocen que hay situaciones que mejorar para considerar el servicio como eficiente; en lo que respecta a la capacidad de respuesta existe una diferencia que se contrapone a lo que los directivos esperaban del servicio, donde los estudiantes resaltan la importancia de mantener canales de comunicación efectivos que faciliten el flujo de la información durante todo el periodo de nivelación; la dimensión de empatía también refleja una brecha distinta a lo que los directivos del CPAl esperaban que percibieran sus estudiantes, lo cual evidencia el compromiso que existe por parte de los colaboradores 
del CPAI para la presentación del servicio, sin embargo, aún existen debilidades que son percibidas por los estudiantes y que inciden en el nivel de calidad con el que valoran el proceso de nivelación, las que se reflejan en el posterior diagrama Ishikawa.

\section{Fase 3. Análisis causas como} base para la mejora

Los resultados evidenciados a través de la aplicación de encuestas a estudiantes y directivos del Centro de Promoción y Apoyo al Ingreso (CPAI) de la Universidad Técnica de Manabí demuestran que en términos generales el nivel de percepción de calidad es alto, sin embargo, cuando se comparó los resultados de la percepción de estudiantes y directivos con respecto al servicio se presentaron diferencias que, aunque no son tan significativas, promueven la mejora continua.

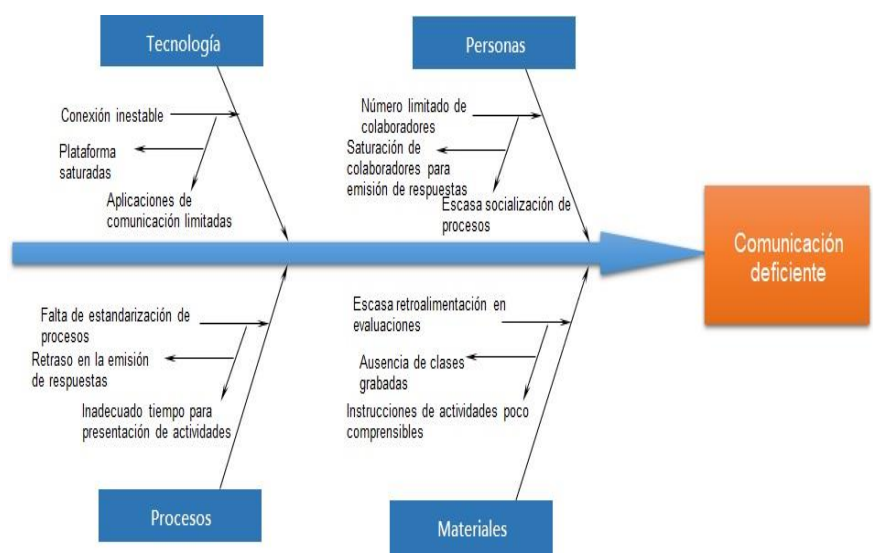

Figura 1. Diagrama de Ishikawa basado en la diferencia 1. Elaborado por: Autoría Propia
De esta manera se pude apreciar que la debilidad en cuanto a la prestación del servicio radica en la comunicación en los diferentes aspectos como el tecnológico, cuando de tecnificar el servicio de trata, sobre todo, vinculado a situaciones de conectividad, aplicaciones y el colapso de plataformas tecnológicas donde se desarrolla el proceso enseñanza aprendizaje.

Según Araiza, Zambrano \& Ramírez (2016) la satisfacción del estudiante proviene del campo de la gestión, en tal sentido, las IES deben entender que la mejora de la calidad no se produce únicamente por incrementar sus recursos e infraestructura, sino por la aportación que hace el capital humano a la sociedad; en tal sentido, las personas juegan un papel importante dentro de cualquier empresa y más aún en aquellas vinculadas a la educación como es el caso del CPAI, donde de acuerdo a la apreciación de los estudiantes es necesario incrementar el número de colaboradores para agilizar el proceso comunicativo, ya que la respuesta es lenta y en la mayoría de las situaciones, afecta al rendimiento académico de los estudiantes. 
En lo que respecta a los procesos, estos carecen de estandarización y uniformidad, lo cual hace que los estudiantes presenten inconvenientes en el momento de requerir algún tipo de información, discrepancias entre los diferentes usuarios, así como asignación de tiempos fuera de la valoración previa de una actividad. Pulido (2017) sostiene que, los procesos de admisión deben de ser previamente socializados, monitoreados y evaluados para lograr ser más eficientes y cumplir con los objetivos organizacionales. En cuanto a los materiales, los estudiantes manifiestan que existe escasa retroalimentación cuando rinden evaluaciones, no cuentan con las clases grabadas para su posterior revisión y en ocasiones, existen actividades donde las instrucciones para el desarrollo de estas se tornan confusas, sin que exista respuesta inmediata para resolver inquietudes al respecto.

En dicho contexto, se justifica la razón por la cual existe la mayor brecha en la dimensión de empatía, donde por más predisposición que exista por parte de los colaboradores para atender a los estudiantes, existen factores internos como los problemas suscitados en la plataforma que se presentan en toda la comunidad universitaria; y externos como la reducción de asignaciones presupuestarias para las IES, lo que las ha obligado a optimizar su recurso humano, disminuyendo la calidad del servicio en cuanto a la capacidad de respuesta, ante lo cual Martín (2019) recomienda definir tiempos específicos destinados a trabajar con los estudiantes para desarrollar actividades que faciliten el proceso de enseñanza - aprendizaje y que incluso permita resolver dudas 0 recabar sugerencias que promuevan la mejora continua y por ende la calidad del servicio.

\section{Conclusiones}

La calidad es una característica necesaria en todos los ámbitos de la sociedad, por cuanto es la garantía de que se proporcionan productos que satisfacen las necesidades de los clientes. El presente trabajo desarrolló un estudio destinado a evaluar la percepción de la calidad del proceso de nivelación del Centro de Promoción y Apoyo al Ingreso 
(CPA) de la Universidad Técnica de Manabí, ante lo cual se concluye lo siguiente:

Los estudiantes en términos generales se encuentran satisfechos con el servicio de Nivelación proporcionado por el CPAI, resaltando la importancia de la empatía en el proceso 2020-S1 que se desarrolló en su totalidad en forma virtual.

El análisis de la diferencia 1 propuesta por el modelo SERVQUAL permitió identificar las brechas que existen entre la percepción de los estudiantes y la de los directivos, las cuales se suscitan principalmente por la falta de recursos para mejorar los servicios técnicos y tecnológicos que faciliten la comunicación y el proceso de enseñanza-aprendizaje; dejando claro incluso que "calidad" se asocia a percepción y subjetividad de los actores educativos.

La comunicación deficiente fue una limitante que se pudo identificar a través del análisis de la brecha 1 y que reveló las causas vinculadas a la tecnología, personas, procesos y materiales. Es importante que las IES dediquen los recursos y el tiempo necesario para monitorear y evaluar su gestión.

\section{Bibliografía}

Álvarez, J., Chaparro, E., \& Reyes , D. (2014). Estudio de satisfacción de los estudiantes con los servicios educativos brindados por Instituciones de Educación Superior del Valle de Toluca. Obtenido de https://revistas.uam.es/reice/ article/view/2788/3003

Araiza , M., Zambrano , A., \& Ramírez , J. (2016). Calidad de los servicios universitarios: Una percepción desde sus actores en una escuela de negocios. Obtenido de https://core.ac.uk/download/p df/84813014.pdf

Barahona, E., \& Ferrada, R. (2012). Medición de calidad de servicio de la Facultad de Ciencias Empresariales de la Universidad del BIO-BIO. Obtenido de http://repobib.ubiobio.cl/jspui/ bitstream/123456789/758/1/F errada\%20Andrade\%2C\%20 Rocio.pdf

Basantes, R. (2017). Modelo SERVQUAL académico como factor de desarrollo de la calidad de los servicios educativos y su influencia en la satisfacción de los estudiantes de las carreras profesionales de la Universidad Nacional Chimborazo Riobamba Ecuador. Obtenido de http://cybertesis.unmsm.edu. 
pe/bitstream/handle/20.500.1 2672/6132/Basantes_ar.pdf? sequence $=1$ \&is Allowed $=y$

Bustamante, M., Zerda, E., Obando, F., \& Tello, M. (2019). Fundamentos de calidad de servicio, el modelo Servqual. Obtenido de https://dialnet.unirioja.es/servl et/articulo?codigo $=7361518$

Cárdenas, A. (2016). Calidad en la gestión universitaria ecuatoriana: Una búsqueda latente de eficiencia educativa. Multiciencias, 16 (2), 194-201.

CPAI. (2020). Consolidado 2020 S1 Número de estudiantes por carrera. Portoviejo: Universidad Técnica de Manabí.

Delgado, A., Santillán, J., Japón, A., \& Mora, B. (2018). Percepciones de los Aspirantes sobre el Proceso de Admisión a la Universidad Pública Ecuatoriana. INNOVA Research Journal, Vol 3, No. 10, 77-90.

Matas , A. (2018). Diseño del formato de escalas tipo Likert: un estado de la cuestión. Obtenido de http://www.scielo.org.mx/sciel o.php?script=sci_arttext\&pid= S1607-40412018000100038

Matsumoto, R. (2014). Desarrollo del Modelo Servqual para la medición de la calidad del servicio en la empresa de publicidad Ayuda Experto. Obtenido de http://www.scielo.org.bo/sciel o.php?pid=S1994-

$37332014000200005 \&$ script $=$ sci_arttext\&tIng=en

Morilla , V. (2019). Valores y empatía. Un estudio en la educación secundaria. Obtenido de http://repositori.uji.es/xmlui/bit stream/handle/10234/183074 /TFM_2019_MartinMorilla_Vi ctor.pdf?sequence $=1$ \&isAllow ed $=y$

Organización de las Naciones Unidas para la Educación, la Ciencia y la Cultura [UNESCO]. (1996). Conferencia Regional sobre Políticas y Estrategias para la Transformacion de la Educación Superior en América Latina y el Caribe. La Habana, Cuba: UNESCO, CRESALC, Ministerio de Educación Superior de la República de Cuba.

Parasuraman, A., Zeithaml, V., \& Berry, L. (1994). "Alternative Scales for Measuring Service Quality: A Comparative Assessment Based on Psychometric and Diagnostic Criteria". Journal of Retailing, 70, 201-230.

Pesantez, F. (2011). Indicadores de gestiñon y calidad en la educación superior. un modelo de evaluación para la Universidad Politécnica 


\section{Salesiana. Quito : Universidad Politécnica Salesiana.}

Pulido, C. (2017). Propuesta de estandarización de los procesos de admisión y de formación académica para la Fundación Piccolino. Obtenido de https://repository.ucatolica.ed u.co/bitstream/10983/15622/1 /PROPUESTA\%20DE\%20ES TANDARIZACI\%C3\%93N\%2 ODE\%20LOS\%20PROCESO S\%20DE\%20ADMISI\%C3\%9 3N\%20Y\%20DE\%20FORMA Cl\%C3\%93N\%20ACAD\%C3 \%89MICA\%20PARA\%20LA \%20FUNDACI.pdf

Sáinz, J., \& Barberá, R. (2019). Dignóstico de la educación superior en Iberoamérica. Madrid: Organización de Estados Iberoamericanos para la Educación, la Ciencia y la Cultura (OEI).

Sánchez, J., Chávez, J., \& Mendoza, J. (2018). LA CALIDAD EN LA EDUCACIÓN SUPERIOR: UNA MIRADA AL PROCESO DE EVALUACIÓN $Y$ ACREDITACIÓN DE UNIVERSIDADES DEL ECUADOR. Revista: Caribeña de Ciencias Sociales, ISSN 2254-7630.

SENESCYT. (2019). Reglamento del Sistema Nacional de Nivelación y Admisión SNNA. Obtenido de http://admision.senescyt.gob. ec/media/2019/05/REGLAME
NTO-SNNA_-Acuerdo-N--2019-030.pdf

Torche, P., Martínez, J., Madrid, J., \& Araya , J. (2015). ¿Qué es la educación de calidad para directores y docentes? Obtenido de https://scielo.conicyt.cl/scielo. php?script=sci_arttext\&pid=S 0718-45652015000200004 